\title{
Spectroscopic Identification of the Au-C Bond Formation upon Electroreduction of an Aryl Diazonium Salt on Gold
}

\author{
Limin Guo, ${ }^{\dagger}, \S$ Lipo Ma, ${ }^{\dagger}$ Yelong Zhang, ${ }^{\dagger}$, Xun Cheng, ${ }^{\#}$ Ye Xu, ${ }^{\#}$ Jin Wang, ${ }^{\dagger, t}$ Erkang \\ Wang,,$^{+*}$ and Zhangquan Peng, ${ }^{\dagger}, *$
}

${ }^{\dagger}$ State Key Laboratory of Electroanalytical Chemistry, Changchun Institute of Applied Chemistry, Chinese Academy of Sciences, Changchun, Jilin 130022, China

\section{"College of Physics, Jilin University, Changchun, Jilin 130012, China \\ ${ }^{\S}$ University of Chinese Academy of Sciences, Beijing 100039, China}

\#Department of Chemical Engineering, Louisiana State University, Baton Rouge, Louisiana 70803, USA

\section{Supporting Information}




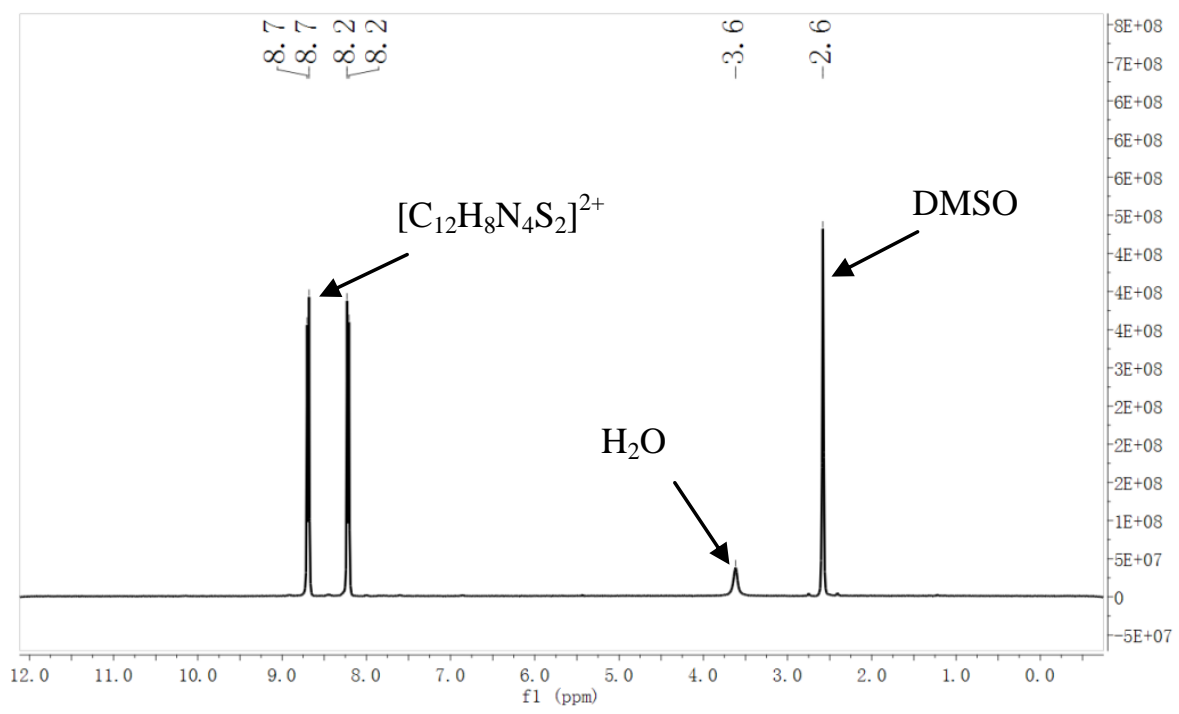

Figure S1. ${ }^{1} \mathrm{H}-\mathrm{NMR}$ characterization of the obtained DSBD in DMSO-d6. 


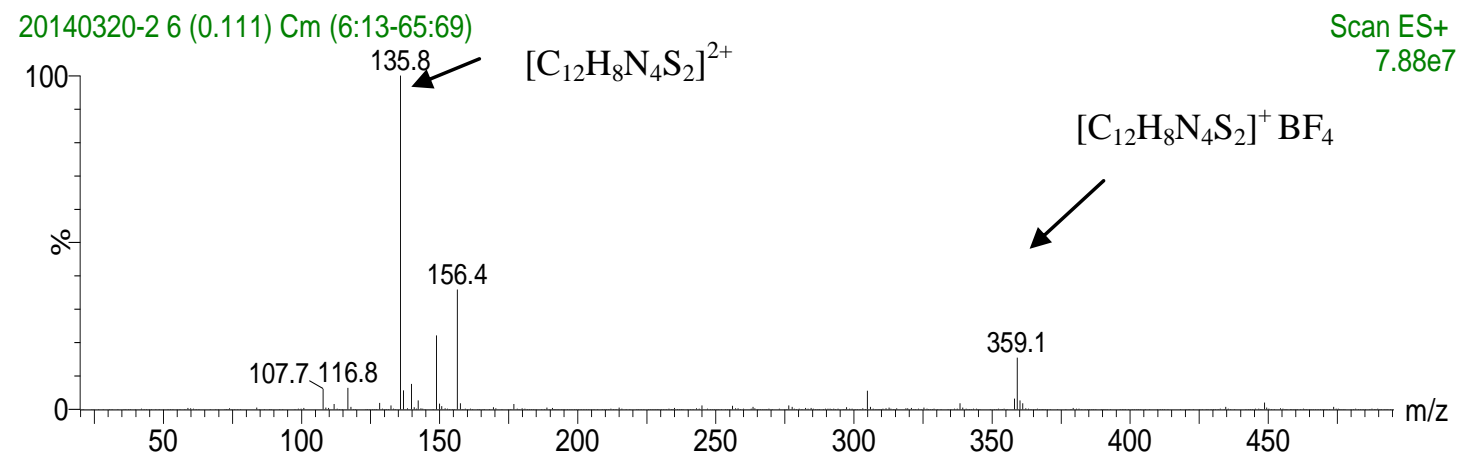

Figure S2. ESI-MS of DSBD in methanol. 


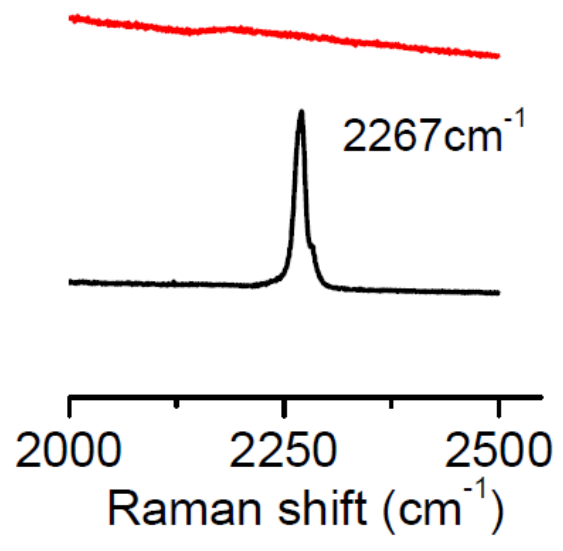

Figure S3. SERS spectra showing the diazonium cations at $\sim 2267 \mathrm{~cm}^{-1}$ (black) of DSBD powder and their absence (red) in the electro-grafted multilayer. 

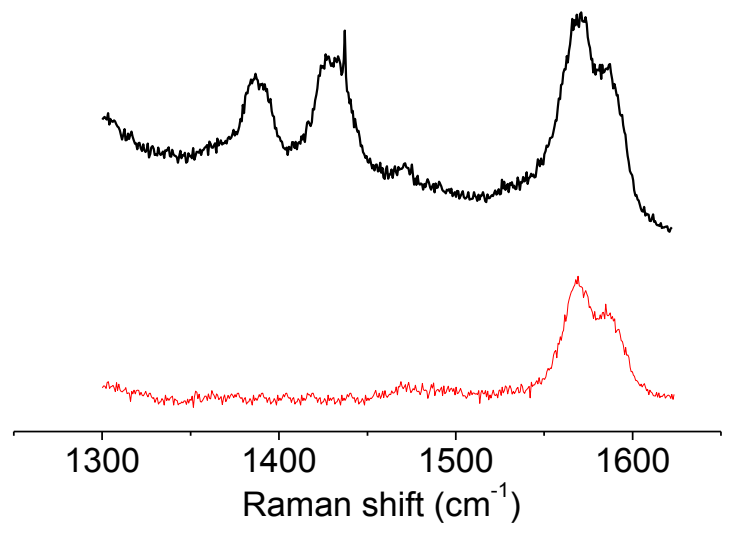

Figure S4. SERS spectra of multilayer (black curve) and monolayer (red curve) showing the loss of $\mathrm{N}=\mathrm{N}$ groups after electrochemical degradation. 


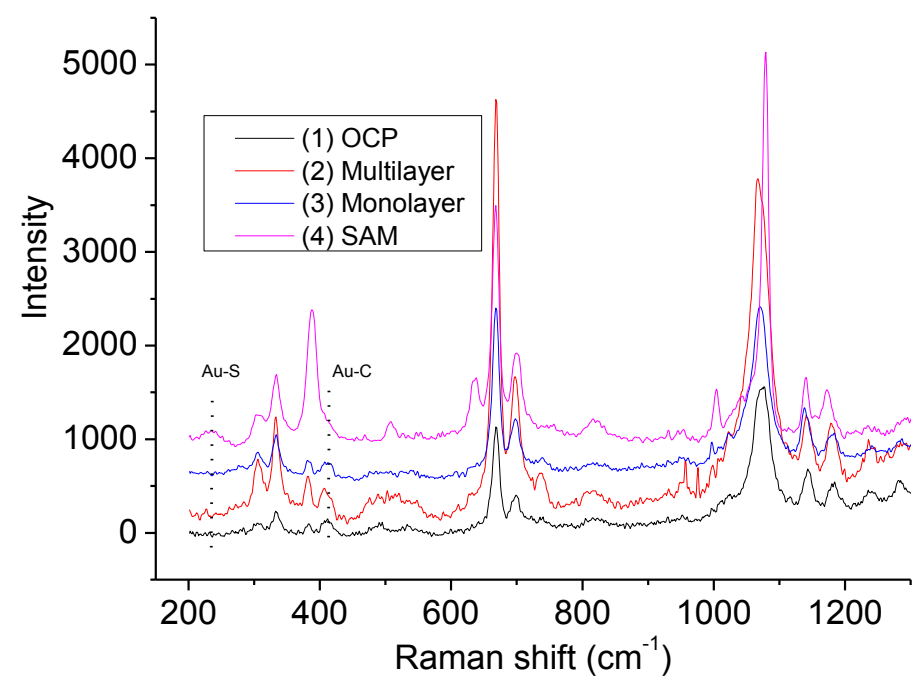

Figure S5. SERS spectra collected on a roughened Au electrode immersed in $0.1 \mathrm{M}$ $\mathrm{TBAClO}_{4} \mathrm{DMSO}$ solution containing $2 \mathrm{mM}$ DSBD (spectrum \# 1); electro-grafted multilayer (spectrum \#2) and monolayer (spectrum \#3), and SAM of bis(4-aminophenyl) disulfide (spectrum \#4) in $0.1 \mathrm{M} \mathrm{TBAClO}_{4} \mathrm{DMSO}$. The Raman bands of Au-S and Au-C were marked with dotted lines. 